\title{
The impact of patient choice on survival in chronic thromboembolic pulmonary hypertension
}

\author{
Syed Rehan Quadery ${ }^{1,2}$, Andrew J. Swift ${ }^{3,4}$, Catherine G. Billings ${ }^{1,2}$, \\ Alfred A.R. Thompson ${ }^{1,2,3}$, Charles A. Elliot ${ }^{1,2}$, Judith Hurdman ${ }^{1,2}$, \\ Athanasios Charalampopoulos ${ }^{1}$, Ian Sabroe ${ }^{1,2,3}$, lain J. Armstrong', \\ Neil Hamilton ${ }^{1}$, Paul Sephton ${ }^{1}$, Sian Garrad ${ }^{1}$, Joanna Pepke-Zaba ${ }^{5}$, \\ David P. Jenkins ${ }^{5}$, Nicholas Screaton ${ }^{5}$, Alexander M. Rothman ${ }^{3}$, Allan Lawrie ${ }^{3}$, \\ Trevor Cleveland 6 , Steven Thomas ${ }^{6}$, Smitha Rajaram ${ }^{6}$, Catherine Hill 6 , \\ Christine Davies $^{6}$, Christopher S. Johns ${ }^{3,6}$, Jim M. Wild ${ }^{3,4}$, Robin Condliffe ${ }^{1,2,7}$ \\ and David G. Kiely ${ }^{1,2,3,4,7}$
}

Affiliations: ${ }^{1}$ Sheffield Pulmonary Vascular Disease Unit, Royal Hallamshire Hospital, Sheffield Teaching Hospitals NHS Foundation Trust, Sheffield, UK. ${ }^{2}$ Academic Directorate of Respiratory Medicine, Sheffield Teaching Hospitals NHS Foundation Trust, Sheffield, UK. ${ }^{3}$ Dept of Infection Immunity and Cardiovascular Disease, University of Sheffield, Sheffield, UK. ${ }^{4}$ Insigneo Institute for in silico Medicine, University of Sheffield, Sheffield, UK. ${ }^{5}$ Pulmonary Vascular Disease Unit, Papworth Hospital NHS Foundation Trust, Cambridge, UK. ${ }^{6}$ Dept of Radiology, Sheffield Teaching Hospitals NHS Foundation Trust, Sheffield, UK. ${ }^{7}$ These authors contributed equally to this work.

Correspondence: David G. Kiely, Sheffield Pulmonary Vascular Disease Unit, Royal Hallamshire Hospital, Sheffield Teaching Hospitals NHS Foundation Trust, Glossop Road, Sheffield, S10 2JF, UK.

E-mail: david.kielydasth.nhs.uk

@ERSpublications

Outcomes for patients undergoing pulmonary endarterectomy are excellent and superior to patients declining surgery http://ow.ly/9UZw30kA28m

Cite this article as: Quadery SR, Swift AJ, Billings CG, et al. The impact of patient choice on survival in chronic thromboembolic pulmonary hypertension. Eur Respir J 2018; 52: 1800589 [https://doi.org/10.1183/ 13993003.00589-2018].

ABSTRACT Pulmonary endarterectomy (PEA) is the gold standard treatment for operable chronic thromboembolic pulmonary hypertension $(\mathrm{CTEPH})$. However, a proportion of patients with operable disease decline surgery. There are currently no published data on this patient group. The aim of this study was to identify outcomes and prognostic factors in a large cohort of consecutive patients with CTEPH.

Data were collected for consecutive, treatment-naive CTEPH patients at the Pulmonary Vascular Disease Unit of the Royal Hallamshire Hospital (Sheffield, UK) between 2001 and 2014.

Of 550 CTEPH patients (mean \pm SD age $63 \pm 15$ years, follow-up $4 \pm 3$ years), $49 \%$ underwent surgery, $32 \%$ had technically operable disease and did not undergo surgery (including patient choice $n=72$ and unfit for surgery $\mathrm{n}=63$ ), and $19 \%$ had inoperable disease due to disease distribution. The 5 -year survival was superior in patients undergoing PEA (83\%) versus technically operable disease who did not undergo surgery $(53 \%)$ and inoperable due to disease distribution $(59 \%)(p<0.001)$. Survival was superior in patients following PEA compared with those offered but declining surgery $(55 \%)(p<0.001)$. In patients offered PEA, independent prognostic factors included mixed venous oxygen saturation, gas transfer and patient decision to proceed to surgery.

Outcomes in CTEPH following PEA are excellent and superior to patients declining surgery, and strongly favour consideration of a surgical intervention in eligible patients.

Copyright CERS 2018. This article is open access and distributed under the terms of the Creative Commons Attribution Licence 4.0. 


\section{Introduction}

Chronic thromboembolic pulmonary hypertension (CTEPH) is a potentially curable form of pulmonary hypertension [1]. It may follow an acute episode of pulmonary embolism [2, 3], but can present as unexplained pulmonary hypertension with no previous history of venous thromboembolism (VTE) [4-6]. CTEPH occurs as a consequence of failure of clot resolution and secondary pulmonary arterial vasculopathy, leading to the development of pulmonary hypertension, right ventricular dysfunction and ultimately death $[5,7]$. Over the past 20 years the treatment of CTEPH has evolved to include pulmonary endarterectomy (PEA) in operable cases [8] and pulmonary hypertension-specific therapy for inoperable disease [9-14]. Balloon pulmonary angioplasty is emerging as a potential treatment option in selected patients with inoperable disease $[1,15,16]$.

PEA is currently considered the treatment of choice in patients with operable CTEPH, and is associated with excellent symptomatic benefit and long-term survival [17-19], with 10-year survival of $72-75 \%$ $[20,21]$. Historical studies in patients treated with anticoagulation alone reported 5-year survival as low as $10 \%$ in patients with a mean pulmonary arterial pressure (mPAP) $>50 \mathrm{mmHg}$ [22, 23]. However, subsequent large registries have shown significant improvements in outcome in a heterogeneous group of nonoperated patients $[24,25]$. Patients with CTEPH may be deemed inoperable where the pulmonary vascular resistance (PVR) is considered to be out of proportion to the degree of surgically accessible obstruction in the pulmonary vasculature [24, 26]. A significant proportion of patients who have technically operable disease do not undergo surgery for a variety of reasons, including comorbidities and patient choice [24-27]. To date, there are only limited data on the clinical course and rationale for treatment decisions in this patient group. In particular, there are very limited data on patients with technically operable disease who have declined surgery.

The aim of the current study was to provide data to help inform patient choice by identifying outcomes and prognostic factors in a large cohort of consecutive patients with CTEPH. Some of the results have been reported previously in abstract form $[28,29]$.

\section{Methods}

Consecutive, treatment-naive patients newly diagnosed with CTEPH at the Pulmonary Vascular Disease Unit of the Royal Hallamshire Hospital (Sheffield, UK) between January 1, 2001 and November 30, 2014 were prospectively recorded in hospital databases, as part of the Sheffield ASPIRE registry (ClinicalTrials.gov identifier NCT02565030). Baseline characteristics, treatment and follow-up data, using a census date of November 30, 2015, were subsequently collected from hospital records and departmental databases. Baseline characteristics, demographics, medical history, pulmonary function, exercise testing, right heart catheter metrics, imaging and treatment were included.

The diagnosis of CTEPH was based on findings obtained after at least 3 months of anticoagulation, and required the patient to have undergone right heart catheterisation and to have $\mathrm{mPAP} \geqslant 25 \mathrm{mmHg}$ at rest and at least one segmental perfusion defect detected by perfusion lung scan or pulmonary artery obstruction seen by multidetector computed tomography angiography or conventional pulmonary angiography with other causes of pulmonary hypertension excluded [18, 19]. Patients were further classified into CTEPH: 1) operated group, 2) technically-operable-not-operated group and 3) patients with nonsurgical disease distribution. The technically-operable-not-operated group was further subclassified into four subgroups based on the reasons for the surgery not being performed: 1) declined surgery (patient choice), 2) being unfit for surgery following multidisciplinary team (MDT) assessment, 3) CTEPH with other contributors to symptoms in addition to clot burden and 4) reason for decision not clear. Suitability for PEA surgery was assessed by a review of clinical and radiological information by the surgical MDT at the national PEA centre at Papworth Hospital (Cambridge, UK).

The date of diagnosis of CTEPH was recorded as the date of the first right heart catheterisation. All patients were followed up until death or the census date of November 30, 2015. Ethical approval was granted for this study (REC 06/Q2308/8).

\section{Statistical analysis}

Descriptive data are presented using mean and standard deviation. Comparisons between groups were made using the t-test (for two groups) and ANOVA (with Bonferroni corrections for three groups) for continuous

This article has supplementary material available from erj.ersjournals.com

The Sheffield ASPIRE registry is registered at ClinicalTrials.gov with identifier NCT02565030.

Received: March 252018 | Accepted after revision: June 152018 
data, and the Chi-squared test for categorical data. Survival was assessed by the Kaplan-Meier method. Comparisons between two groups were performed using the log-rank test. Prognostic variables were assessed using univariate and multivariate Cox regression analysis for survival. 72 variables in the CTEPHsurgical-operated group and 71 variables in the CTEPH-surgical-not-operated and CTEPH-nonsurgical groups were identified based on previous literature $[24,25,30]$ and entered into univariate Cox regression analysis. Variables with a p-value of $<0.20$ at univariate analysis and $<10 \%$ missing data were included for the multivariate analysis using the forward logistic regression method. To allow comparison between continuous variables, hazard ratios (HRs) were scaled using the standard deviation of the variable. A p-value of $<0.05$ was considered statistically significant. Accuracy of variables for prediction of mortality at 3 years was assessed using receiver operator characteristic (ROC) curve analysis. Statistical analysis was performed using SPSS version 25 (IBM, Armonk, NY, USA) and Prism software (GraphPad, La Jolla, CA, USA).

550 patients with CTEPH (mean \pm SD age $63 \pm 15$ years, $50 \%$ female) were identified. A flowchart giving the classification with a detailed breakdown of patients is shown in figure 1.

\section{Results}

\section{Patient characteristics}

CTEPH (whole cohort)

Baseline characteristics for the major groups are summarised in table 1 and for technically-operablenot-operated subgroups in supplementary table S1. There was excellent data completeness, with data on lung function testing, exercise testing and imaging available in $94 \%, 94 \%$ and $93 \%$ of patients, respectively. Right heart catheterisation was performed in all patients. Survival data to the census date were available for all patients. The most common presenting symptoms were breathlessness (98\%), ankle swelling (38\%), pre-syncope (27\%) and chest pain (19\%). There was no significant difference in symptoms or duration of symptoms prior to diagnosis between patients with technically operable disease undergoing and not undergoing surgery and those with a nonsurgical disease distribution. In terms of ethnicity, $95 \%$ of patients were White, with no significant difference in ethnicity between the CTEPH groups. With respect to

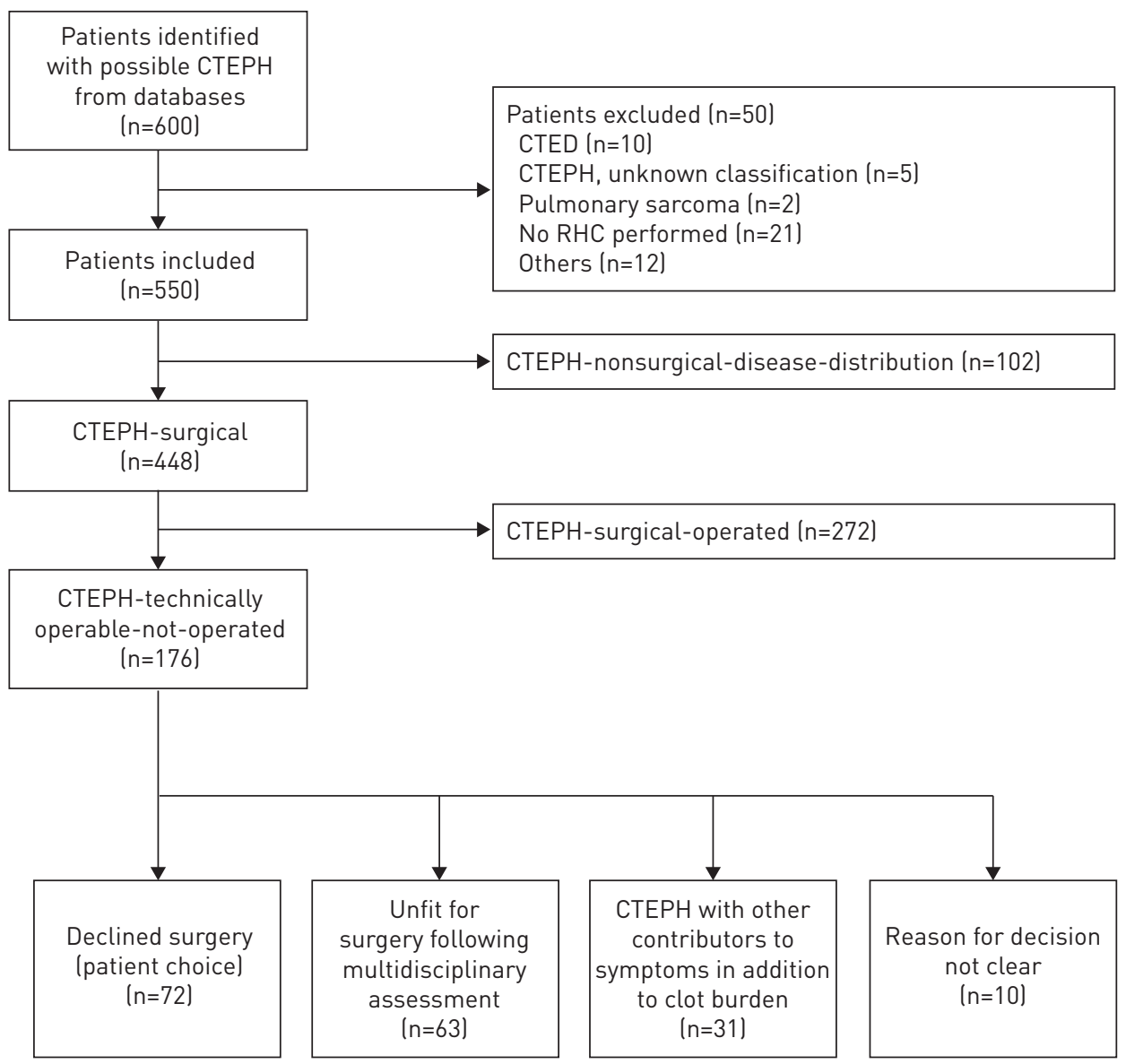

FIGURE 1 Patient cohort flowchart showing group/subgroup classification. CTEPH: chronic thromboembolic pulmonary hypertension; CTED: chronic thromboembolic disease; RHC: right heart catheterisation. 
TABLE 1 Baseline characteristics of patients with chronic thromboembolic pulmonary hypertension (CTEPH)

\begin{tabular}{|c|c|c|c|c|}
\hline & $\begin{array}{l}\text { CTEPH (whole } \\
\text { cohort) }\end{array}$ & $\begin{array}{l}\text { CTEPH-operated } \\
\text { group }\end{array}$ & $\begin{array}{c}\text { CTEPH-technically-operable- } \\
\text { not-operated group }\end{array}$ & $\begin{array}{c}\text { CTEPH-nonsurgical-disease- } \\
\text { distribution group }\end{array}$ \\
\hline Subjects & $550(100)$ & 272 (49) & $176(32)$ & 102 (19) \\
\hline Female & 50 & $45^{+, 9}$ & $50^{\#}$ & $62^{\#}$ \\
\hline $\mathrm{BMI} \mathbf{k g} \cdot \mathrm{m}^{-2}$ & $29 \pm 7$ & $30 \pm 7$ & $29 \pm 8$ & $29 \pm 6$ \\
\hline \multicolumn{5}{|l|}{ Duration of symptoms } \\
\hline$<1$ year & 15 & 14 & 17 & 12 \\
\hline$>5$ years & 16 & 16 & 14 & 18 \\
\hline Not clear & 8 & 3 & 14 & 9 \\
\hline WHO FC I/II versus III/IV & $11 / 89$ & $13 / 87^{+}$ & $11 / 89$ & $3 / 97^{\#}$ \\
\hline ISWD m & $189 \pm 177$ & $232 \pm 185^{\uparrow,+}$ & $142 \pm 157^{\#}$ & $155 \pm 160^{\#}$ \\
\hline RAP mmHg & $11 \pm 5$ & $11 \pm 5$ & $11 \pm 6$ & $12 \pm 5$ \\
\hline mPAP mmHg & $46 \pm 11$ & $47 \pm 11^{\uparrow}$ & $43 \pm 11^{\#,+}$ & $48 \pm 12^{9}$ \\
\hline FEV $1 \%$ pred & $80 \pm 35$ & $83 \pm 43^{9}$ & $75 \pm 23^{\#}$ & $80 \pm 24$ \\
\hline FVC $\%$ pred & $92 \pm 24$ & $95 \pm 21$ & $89 \pm 25$ & $90 \pm 27$ \\
\hline DLco \% pred & $61 \pm 17$ & $65 \pm 15^{9}$ & $55 \pm 19^{\#,+}$ & $61 \pm 17^{9}$ \\
\hline History of acute VTE & 71 & $74^{+}$ & $74^{+}$ & $57^{\#, 9}$ \\
\hline IVC filter & 3 & 3 & 5 & 1 \\
\hline Thrombophilia & 5 & 6 & 3 & 3 \\
\hline History of cancer & 11 & $6^{\Uparrow,+}$ & $15^{\#}$ & $15^{\#}$ \\
\hline Smoking & 38 & 38 & 43 & 30 \\
\hline Obesity & 36 & 39 & 32 & 32 \\
\hline Splenectomy & 5 & $3^{+}$ & $3^{+}$ & $12^{\#, q}$ \\
\hline Thyroid disorder & 12 & $8^{+}$ & 14 & $18^{\#}$ \\
\hline VA shunt/PPM infection & 2 & 2 & 0 & 4 \\
\hline
\end{tabular}

Data are presented as $\mathrm{n}(\%)$, mean \pm SD or \%. BMI: body mass index; WHO FC: World Health Organization Functional Class; ISWD: incremental shuttle walk distance; RAP: right atrial pressure; mPAP: mean pulmonary arterial pressure; PCWP: pulmonary capillary wedge pressure; PVR: pulmonary vascular resistance; WU: Wood units; $\mathrm{SvO}_{2}$ : mixed venous oxygen saturation; FEV1: forced expiratory volume in $1 \mathrm{~s}$; FVC: forced vital capacity; DLCO: diffusing capacity of the lung for carbon monoxide; VTE: venous thromboembolism; IVC: inferior vena cava; VA: ventriculo-atrial; PPM: permanent pacemaker; IBD: inflammatory bowel disease; CAD: coronary artery disease; LV: left ventricular; CKD: chronic kidney disease; COPD: chronic obstructive pulmonary disease; PH: pulmonary hypertension. ${ }^{\#}: p<0.05$ compared with CTEPH-operated; ${ }^{\Uparrow}$ : $p<0.05$ compared with CTEPH-technically-operable-not operated; ${ }^{+}: p<0.05$ compared with CTEPH-nonsurgical-disease-distribution.

anticoagulation, $90 \%$ of the patients received a vitamin $\mathrm{K}$ antagonist, $4.5 \%$ received anti-Xa oral anticoagulants and 5\% received low-molecular-weight heparin, while $0.5 \%$ were not anticoagulated. $76 \%$ of patients received pulmonary hypertension therapy, of whom 315 received phosphodiesterase type 5 inhibitor (PDE-5i) monotherapy, 49 received endothelin receptor antagonist monotherapy, 22 received prostanoid monotherapy, 18 received combination therapy with a PDE-5i and endothelin receptor antagonist, and 12 received combination therapy with a PDE-5i and prostanoid. No patients received riociguat.

\section{CTEPH-operated group}

Patients with CTEPH who underwent surgery were younger with a male predominance, and with better exercise capacity and gas transfer compared with patients in the other groups (table 1). The mean waiting time from date of diagnosis to surgery at the national PEA centre was $290 \pm 175$ days. $74 \%$ received bridging therapy, of whom $74 \%$ received monotherapy with PDE-5i. Bridging therapy was commenced at diagnosis and did not lead to delay in referral for surgical consideration or time to surgery (supplementary table S2). 
CTEPH-technically-operable-not-operated subgroup: declined surgery (patient choice)

Compared with patients undergoing PEA, patients who declined surgery were older, more likely to be female, and had more comorbidities and lower exercise capacity (all p<0.05) (table 2).

\section{CTEPH-technically-operable-not-operated subgroup: not offered surgery lunfit for surgery, other} contributors to symptoms in addition to clot burden]

Patients who were unfit for surgery had worse exercise capacity, lower diffusing capacity of the lung for carbon monoxide (DLCO), were more likely to be current smokers and had a higher prevalence of chronic obstructive pulmonary disease (COPD) than other technically operable patients not undergoing surgery. Patients with other contributors to symptoms in addition to clot burden (where the risk of surgery was felt to outweigh the benefit) had milder pulmonary haemodynamics and a better exercise capacity than the other two subgroups. The baseline characteristics of these patients are described in further detail in supplementary table S1.

\section{CTEPH-nonsurgical-disease-distribution group}

History of VTE was less common in patients with nonsurgical disease. A history of thyroid disease and splenectomy was also significantly more common in this subgroup compared with patients undergoing PEA (table 1).

\section{TABLE 2 Baseline characteristics of chronic thromboembolic pulmonary hypertension (CTEPH)-operated group versus patients who declined surgery (patient choice)}

\begin{tabular}{|c|c|c|c|}
\hline & CTEPH-operated & Declined surgery (patient choice) & p-value \\
\hline Subjects n & 272 & 72 & \\
\hline Age years & $58 \pm 14$ & $68 \pm 16$ & $<0.001$ \\
\hline Female & 45 & 63 & 0.007 \\
\hline $\mathrm{BMI} \mathrm{kg} \cdot \mathrm{m}^{-2}$ & $30 \pm 7$ & $29 \pm 7$ & 0.485 \\
\hline WHO FC I/II versus II/IV & $13 / 87$ & $17 / 83$ & 0.462 \\
\hline Duration of symptoms & & & 0.009 \\
\hline$<1$ year & 14 & 22 & \\
\hline $1-2$ years & 40 & 38 & \\
\hline $2-5$ years & 27 & 17 & \\
\hline$>5$ years & 16 & 11 & \\
\hline Not clear & 3 & 13 & \\
\hline ISWD m & $232 \pm 185$ & $169 \pm 177$ & 0.009 \\
\hline RAP mmHg & $11 \pm 5$ & $12 \pm 6$ & 0.192 \\
\hline mPAP mmHg & $47 \pm 11$ & $46 \pm 10$ & 0.360 \\
\hline Cardiac index $L \cdot \min ^{-1} \cdot \mathrm{m}^{-2}$ & $2.5 \pm 0.8$ & $2.6 \pm 0.8$ & 0.305 \\
\hline PCWP mmHg & $12 \pm 4$ & $12 \pm 5$ & 0.667 \\
\hline PVR WU & $7.7 \pm 4$ & $8 \pm 4$ & 0.767 \\
\hline $\mathrm{SvO}_{2} \%$ & $61 \pm 8$ & $61 \pm 9$ & 0.610 \\
\hline FEV $1 \%$ pred & $83 \pm 43$ & $82 \pm 21$ & 0.714 \\
\hline FVC $\%$ pred & $95 \pm 21$ & $96 \pm 23$ & 0.702 \\
\hline DLco \% pred & $65 \pm 15$ & $61 \pm 17$ & 0.084 \\
\hline History of VTE & 74 & 69 & 0.489 \\
\hline History of cancer & 6 & 6 & 0.827 \\
\hline Smoking & 38 & 35 & 0.664 \\
\hline Obesity & 39 & 26 & 0.048 \\
\hline CAD & 11 & 8 & 0.620 \\
\hline LV dysfunction & 2 & 6 & 0.131 \\
\hline CKD & 3 & 14 & $<0.001$ \\
\hline COPD & 13 & 17 & 0.356 \\
\hline $\mathrm{PH}$ therapy following diagnosis & 74 & 75 & 0.849 \\
\hline
\end{tabular}

Data are presented as mean \pm SD or $\%$, unless otherwise stated. BMI: body mass index; WHO FC: World Health Organization Functional Class; ISWD: incremental shuttle walk distance; RAP: right atrial pressure; mPAP: mean pulmonary arterial pressure; PCWP: pulmonary capillary wedge pressure; PVR: pulmonary vascular resistance; WU: Wood units; $\mathrm{SvO}_{2}$ : mixed venous oxygen saturation; $\mathrm{FEV} 1$ : forced expiratory volume in $1 \mathrm{~s}$; FVC: forced vital capacity; DLCO: diffusing capacity of the lung for carbon monoxide; VTE: venous thromboembolism; CAD: coronary artery disease; LV: left ventricular; CKD: chronic kidney disease; COPD: chronic obstructive pulmonary disease; $\mathrm{PH}$ : pulmonary hypertension. Comparisons between continuous variables and categorical variables were made by the t-test and the Chi-squared test, respectively. 


\section{Outcomes}

\section{CTEPH (whole cohort)}

During a mean \pm sD follow-up of $4 \pm 3$ years, 182 (32\%) patients died: 51 in the CTEPH-surgical-operated group, 76 in the CTEPH-technically-operable-not-operated group and 49 in the CTEPH-nonsurgicaldisease-distribution group. Estimated 1-, 3- and 5-year survival from date of right heart catheterisation was superior in the CTEPH-operated group (97\%, 87\% and $83 \%$, respectively) compared with both the CTEPH-technically-operable-not-operated group $(87 \%, 63 \%$ and $53 \%$, respectively) and CTEPHnonsurgical-disease-distribution group $(92 \%, 75 \%$ and $59 \%$, respectively) $(\mathrm{p}<0.001)$ (figure 2 ).

\section{CTEPH-operated group}

Persistent pulmonary hypertension (defined by $\mathrm{mPAP} \geqslant 25 \mathrm{mmHg}$ ) post-PEA surgery was found in 108 (40\%) patients in our study. There was no significant difference in long-term survival between the patients who developed pulmonary hypertension post-PEA versus the patients who did not develop pulmonary hypertension post-PEA ( $p=0.288$ ). However, those with a post-operative PVR above the median ( 3 Wood units (WU)) had a worse prognosis following surgery $(\mathrm{p}=0.013)$. There was no significant difference in survival between patients who received bridging therapy versus patients who did not receive bridging therapy prior to surgery (3-year survival $86 \%$ versus $90 \%$; $\mathrm{p}=0.447$ ), although those who received bridging therapy had more severe pulmonary haemodynamics at diagnosis (mPAP 50 versus $40 \mathrm{mmHg}$ and PVR 8.8 versus 5 WU; both $\mathrm{p}<0.001$ ).

\section{CTEPH-technically-operable-not-operated subgroup: declined surgery (patient choice)}

The estimated 5-year survival of patients declining surgery was significantly worse than those undergoing PEA (55\% versus $83 \%$; $\mathrm{p}<0.001$ ) (figure $3 \mathrm{a}$ ). The impact of age on long-term outcome in patients offered surgery is shown in figure $3 b-d$. A survival benefit was seen in patients aged $<60$ years $(p=0.036)$ and $\geqslant 60$ years $(p<0.001)$ (figure $3 b$ and $c$, respectively). A trend in favour of surgery was observed in more elderly patients aged $\geqslant 70$ years $(p=0.056)$ (figure $3 d)$.

\section{CTEPH-technically-operable-not-operated subgroup: not offered surgery lunfit for surgery, other} contributors to symptoms in addition to clot burden)

Patients deemed unfit for surgery had a significantly worse survival than patients offered surgery who declined, who had a significantly worse survival than patients with other contributors to symptoms in addition to clot burden $(\mathrm{p}<0.05)$ (supplementary figure $\mathrm{S} 1$ ).

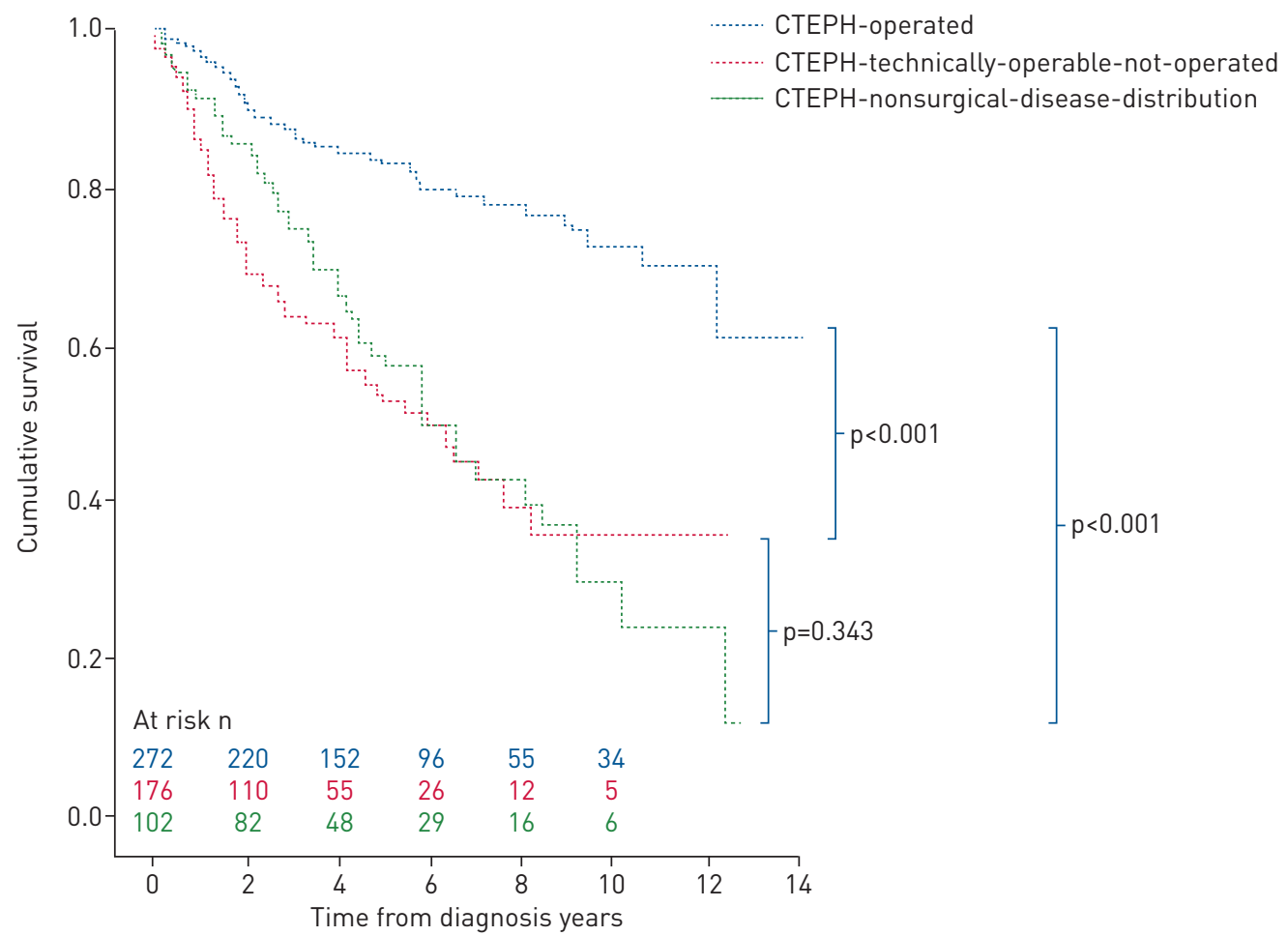

FIGURE 2 Kaplan-Meier estimates of survival from date of diagnosis in the chronic thromboembolic pulmonary hypertension (CTEPH)-operated, CTEPH-technically-operable-not-operated and CTEPH-nonsurgical patient groups. 

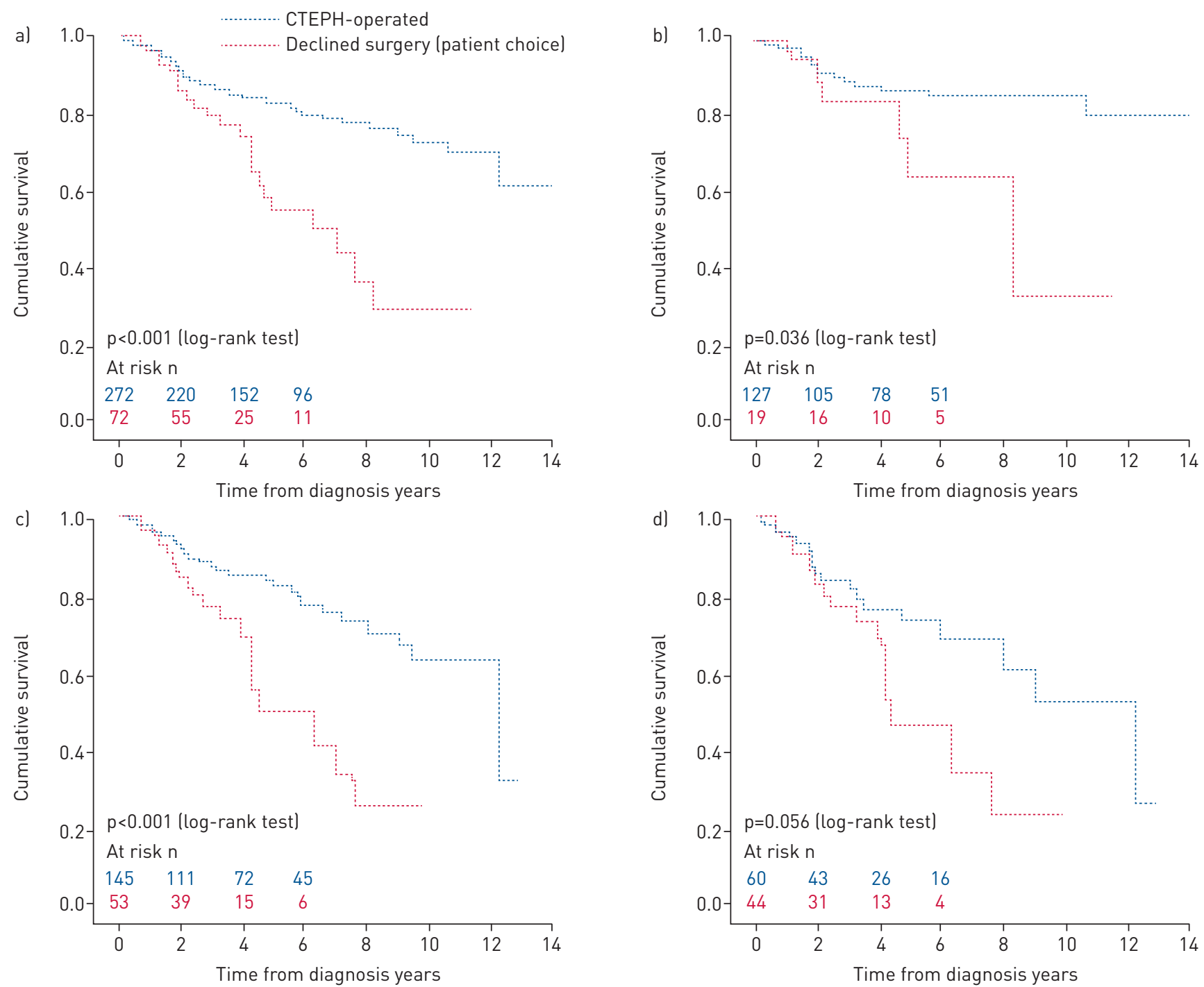

FIGURE 3 Kaplan-Meier estimates of survival from date of diagnosis comparing outcomes in patients with technically operable chronic thromboembolic pulmonary hypertension (CTEPH) who were offered surgery and underwent pulmonary endarterectomy versus patients who declined surgery (patient choice): a) all patients, b) patients $<60$ years, c) patients $\geqslant 60$ years and d) patients $\geqslant 70$ years.

\section{Prognostic indicators \\ CTEPH (whole cohort)}

Univariate analysis of the whole cohort identified a number of predictors of outcome (table 3). Independent predictors of mortality identified from multivariate analysis were: PEA (HR 0.38, 95\% CI 0.23-0.63), DLCO (HR 0.59, 95\% CI 0.46-0.74), mixed venous oxygen saturation $\left(\mathrm{SvO}_{2}\right)(\mathrm{HR} 0.71,95 \% \mathrm{CI}$ 0.57-0.87), history of cancer (HR 2.24, 95\% CI 1.28-3.95), chronic kidney disease (HR 2.20, 95\% CI $1.22-$ 4.71) and age (HR 1.39, 95\% CI 1.06-1.80).

\section{CTEPH-offered-surgery}

Four independent predictors of survival were identified in the combined group of patients offered surgery: patient choice (HR 3.64, 95\% CI 1.95-6.81), $\mathrm{SvO}_{2}$ (HR 0.66, 95\% CI 0.49-0.89), DLCO (HR 0.67, 95\% CI 0.47-0.95) and the presence of coronary artery disease (HR 2.34, 95\% CI 1.11-4.96) (table 4).

CTEPH-technically-operable-not-operated subgroup: declined surgery (patient choice)

ROC curve analysis for prediction of 3-year mortality was performed for the three continuous prognostic variables identified at univariate analysis (supplementary table S3): DLCO (area under the curve (AUC) 0.87), RAP (AUC 0.81) and $\mathrm{SvO}_{2}$ (AUC 0.85). Using median thresholds of DLCO 62\%, RAP $11 \mathrm{mmHg}$ and $\mathrm{SvO}_{2} 62 \%$, the sensitivity, specificity, positive predictive value and negative predictive value for predicting 
TABLE 3 Cox regression survival analysis for chronic thromboembolic pulmonary hypertension (CTEPH) (whole cohort)

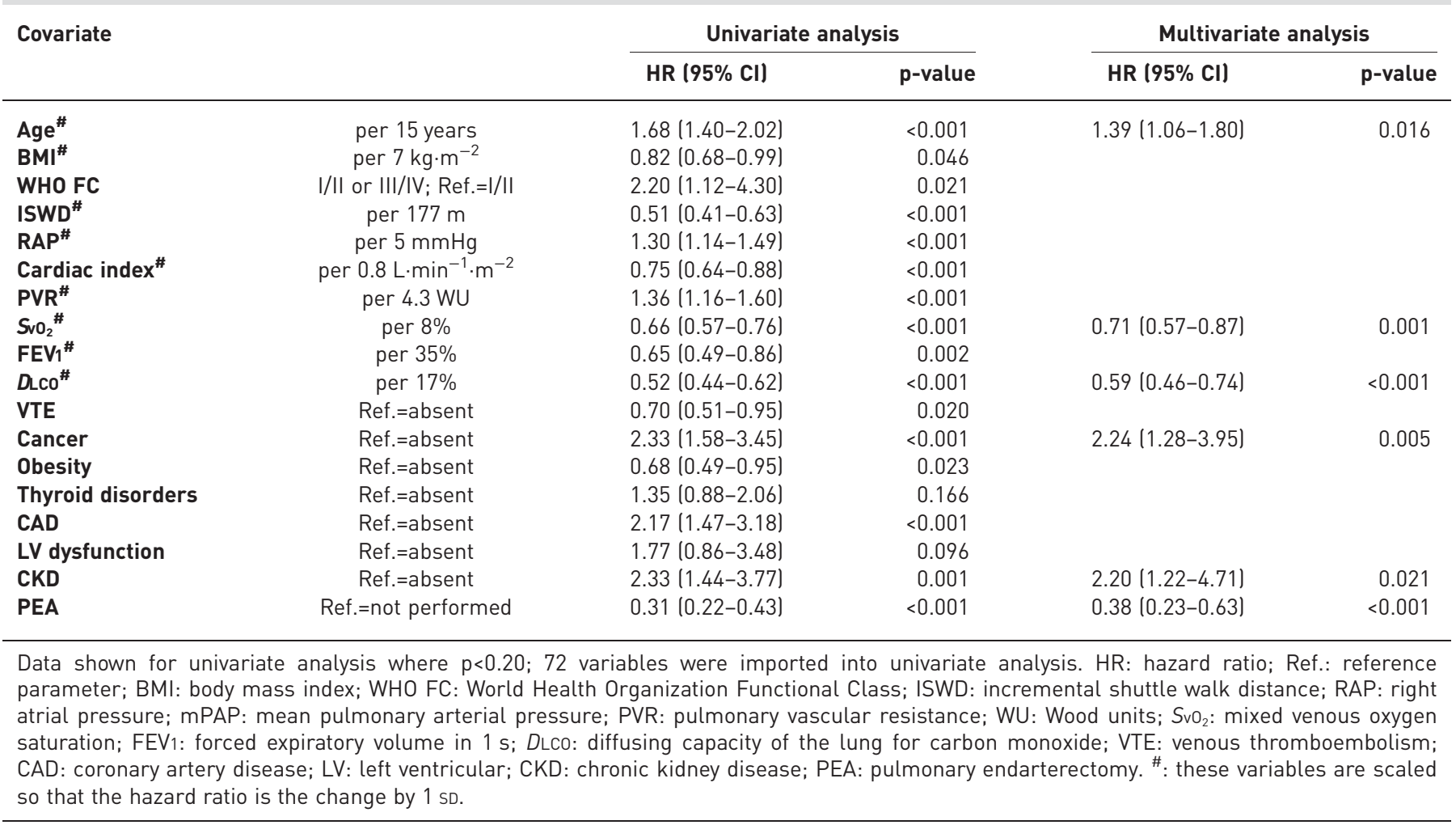

3-year mortality were $100 \%, 63 \%, 31 \%$ and $100 \%, 80 \%, 70 \%, 32 \%$ and $95 \%$, and $90 \%, 60 \%, 30 \%$ and $97 \%$, respectively.

\section{CTEPH-technically-operable-not-operated group}

For the whole group, univariate analysis identified a number of predictors of outcome, including age, World Health Organization Functional Class, exercise capacity, pulmonary haemodynamics and comorbidities. Univariate and multivariate analysis for each of the three subgroups is shown in supplementary table S3. Independent predictors of outcome were $\mathrm{SvO}_{2}(\mathrm{HR} \mathrm{0.53,95 \%} \mathrm{CI} \mathrm{0.38-0.76),} \mathrm{DLCO}$ (HR 0.54, 95\% CI 0.38-0.75) and cancer (HR 4.10, 95\% CI 2.02-8.37). In those who declined surgery, $\mathrm{S}_{\mathrm{vO}}$ (HR 0.24, 95\% CI 0.12-0.51) was an independent predictor of survival; in those unfit for surgery, exercise capacity (HR 0.51, 95\% CI 0.26-0.96), PVR (HR 1.56, 95\% CI 1.02-2.38) and comorbidities (cancer (HR 11.46, 95\% CI 3.46-37.99) and chronic kidney disease (HR 8.87, 95\% CI 2.45-32.17)) independently predicted outcome, while in patients with other contributors to symptoms in addition to clot burden, cancer (HR 9.93, 95\% CI 1.98-49.85) was an independent predictor of outcome.

\section{Discussion}

To the best of our knowledge, this is the first study primarily focusing on patients with technically operable CTEPH who did not undergo PEA. Patient choice, lack of fitness for surgery and the presence of other contributors to symptoms in addition to clot burden were the commonest reasons for patients not undergoing surgery, while pulmonary haemodynamic severity, DLCO and comorbidities were independent predictors of survival. In addition, in a large cohort of consecutive patients with CTEPH we have shown that long-term survival of patients undergoing PEA is excellent and superior to patients declining surgery, strongly favouring consideration of a surgical intervention in eligible patients.

\section{CTEPH (whole cohort)}

PEA is considered the treatment of choice for suitable patients with CTEPH, and is thought to provide the best prospect of improved quality and quantity of life [17-19]. Our study, conducted in a large cohort of consecutive patients with CTEPH, confirms the results of the international CTEPH Registry that PEA is an independent predictor of survival [24]. In operated patients it was associated with an excellent long-term outcome with an estimated 5-year survival of $83 \%$, similar to data from the international [24], 
TABLE 4 Cox regression survival analysis in chronic thromboembolic pulmonary hypertension (CTEPH)-technically-operable who were offered surgery (operated group and declined surgery (patient choice) subgroups)

\begin{tabular}{|c|c|c|c|c|c|}
\hline \multirow[t]{2}{*}{ Covariate } & & \multicolumn{2}{|c|}{ Univariate analysis } & \multicolumn{2}{|c|}{ Multivariate analysis } \\
\hline & & $\operatorname{HR}(95 \% \mathrm{CI})$ & p-value & $\operatorname{HR}(95 \% \mathrm{CI})$ & p-value \\
\hline $\mathrm{Age}^{\#}$ & per 15 years & $1.71(1.30-2.25)$ & $<0.001$ & & \\
\hline Cardiac arrhythmia & Ref.=absent & $2.16(1.03-4.53)$ & 0.043 & & \\
\hline ISWD" & per $185 \mathrm{~m}$ & $0.56(0.41-0.75)$ & $<0.001$ & & \\
\hline RAP $^{\#}$ & per $6 \mathrm{mmHg}$ & $1.57(1.25-1.98)$ & $<0.001$ & & \\
\hline$D \mathrm{LCo}^{\#}$ & per $16 \%$ & $0.56(0.42-0.75)$ & $<0.001$ & $0.67(0.47-0.95)$ & 0.025 \\
\hline VTE & Ref.=absent & $0.62(0.39-0.98)$ & 0.045 & & \\
\hline Cancer & Ref.=absent & $1.77(0.85-3.69)$ & 0.127 & & \\
\hline Obesity & Ref.=absent & $0.55(0.33-10.92)$ & 0.024 & & \\
\hline Thyroid disorders & Ref.=absent & $1.65(0.87-3.15)$ & 0.122 & & \\
\hline CAD & Ref.=absent & $2.21(1.24-3.94)$ & 0.007 & $2.34(1.11-4.96)$ & 0.026 \\
\hline
\end{tabular}

Data shown for univariate analysis where $p<0.20 ; 72$ variables were imported into univariate analysis. HR: hazard ratio; Ref.: reference parameter; WHO FC: World Health Organization Functional Class; ISWD: incremental shuttle walk distance; RAP: right atrial pressure; PVR: pulmonary vascular resistance; WU: Wood units; $\mathrm{SvO}_{2}$ : mixed venous oxygen saturation; DLco: diffusing capacity of the lung for carbon monoxide; VTE: venous thromboembolism; CAD: coronary artery disease; CKD: chronic kidney disease. " : these variables are scaled so that the hazard ratio is the change by 1 sD.

Austrian [31, 32], Spanish [25], Italian [33] and Dutch CTEPH registries [34]. Although 482 patients (82\%) had technically operable disease distribution, despite the proven benefits of PEA only 272 ( $49 \%$ of the total cohort) underwent surgery. Previous registries reported similar proportions of patients who underwent PEA, but provided only limited data on reasons for not undergoing surgery and predictors of long-term outcome $[24,34,35]$.

\section{CTEPH-technically-operable-not-operated group}

176 (39\% of patients with technically operable disease) did not undergo surgery due to patient choice $(n=72)$, concerns regarding fitness to undergo surgery $(n=63)$ or having other contributors to symptoms in addition to clot burden ( $n=31$ where the benefits of surgery were felt to be minimal). The 5 -year survival in patients with technically accessible disease not undergoing surgery was $53 \%$, significantly better than historical studies of patients with CTEPH treated with anticoagulation alone $[22,23]$. Survival was related to the rationale underpinning the treatment decision, with those declining surgery having a superior survival to those who were deemed unfit for surgery. Not only did markers of disease severity such as $\mathrm{SvO}_{2}$ and DLCO independently predict survival, but also the presence of comorbidities, emphasising the impact of conditions apart from the pulmonary vasculature when making treatment decisions.

\section{CTEPH-technically-operable-not-operated subgroup: declined surgery (patient choice)}

The proportion of patients with technically operable disease who were offered surgery $(\mathrm{n}=344)$ but declined $(n=72(21 \%))$ is larger than previously noted in other registries and may reflect the consecutively enrolled nature of our study $[24,34,35]$. These data highlight the importance of patients being referred for assessment and counselling by a PEA surgeon and experienced MDT. In patients who declined surgery the severity of pulmonary haemodynamics and DLCO predicted outcome, with median thresholds for DLCO, right atrial pressure and $\mathrm{S}_{\mathrm{VO}_{2}}$ having negative predictive values for 3-year mortality $>95 \%$. For a selected cohort of patients who despite counselling decline surgery this information may be useful, although it must also be emphasised that quality of life benefits in the majority of patients are greater with surgery [36, 37]. In contrast, patients with severe haemodynamics assessed to be good surgical candidates may find data highlighting a poor prognosis in the absence of a surgical intervention an aid to decision making.

This study has demonstrated a significantly superior survival in patients with CTEPH who were offered surgery and underwent PEA compared with those who declined. Although patients declining surgery were older, with a poorer exercise capacity and more comorbidities, declining surgery due to patient choice was 
an independent predictor of a worse outcome. In patients who declined compared with those who underwent surgery, there was a female predominance [27] and sex-specific factors related to risk-taking may play a role [38]. Given the findings of this study and the benefit of PEA, more work is required to understand the factors underlying decisions to decline surgery.

\section{Unfit for surgery}

In expert hands PEA has a perioperative mortality of $<5 \%$ and offers the best chance of longer-term survival, but requires careful assessment of risks versus benefits for individual patients [21, 24]. We found 63 patients (14\% of those with technically operable disease) were deemed unfit for surgery by the MDT. These patients had a significantly poorer survival than those declining surgery. Alternative interventions to surgery such as balloon pulmonary angioplasty may appear attractive in these patients, but the presence of significant comorbidities may be primary determinants of survival. A meticulous assessment balancing the potential symptomatic benefit versus the risks of such interventions is therefore paramount where mortality benefit is not clear.

\section{CTEPH-nonsurgical-disease-distribution group}

$19 \%$ of all patients were deemed to have nonsurgical disease distribution. This proportion is similar to the international CTEPH Registry (20\%) [24], but less than the UK (32\%) [35] and Dutch (26\%) [34] registries. This may reflect an increasing willingness among surgeons to operate on patients with disease that would previously have been considered too distal to benefit from surgery. Indeed, a number of centres have shown that outcomes in patients with type 3 disease (more distal) in expert hands are now similar to more proximal disease (type 1 and 2) [21,39]. We noted a female predominance, an increased incidence of thyroid disease and splenectomy, and reduced incidence of VTE in this group (table 1), in keeping with previous reports [33].

\section{CTEPH-operated group: timing of surgery and pulmonary vasodilator therapy}

The mean \pm SD time from diagnosis to PEA surgery was $290 \pm 175$ days, longer than in previously reported studies $[24,32,35,40]$, but in line with waiting times for surgery in the UK during the duration of this study, although UK waiting times are now falling. In keeping with data from the international CTEPH Registry, the duration of delay did not impact on long-term survival [24]. A large number of patients were bridged to PEA with off-label pulmonary arterial hypertension-specific therapies (74\%). Although there is no published evidence to support this practice, this may reflect the longer time from referral to surgery in the UK during the study period compared with that reported in the international CTEPH Registry [24]. Importantly, bridging therapy had no effect on time to referral or to surgery. Patients receiving bridging therapy in the present study had similar haemodynamics to those who received bridging therapy in the international CTEPH Registry, while patients who were not bridged to surgery had milder haemodynamics than those in the international CTEPH Registry [24]. Importantly, in our study receiving bridging therapy was not associated with adverse outcome at univariate analysis.

\section{Limitations}

This study pre-dates the availability of balloon angioplasty and riociguat therapy in the UK, and therefore the impact of these interventions and their potential benefits cannot be assessed. Although patient-specific data were enriched by retrospective case note review and interrogation of databases, this resulted in higher levels of data completeness than in other contemporary registries. Furthermore, the consecutive nature of enrolment in the ASPIRE registry reduces recruitment bias associated with previous nonconsecutively enrolled studies. This study provides no data on the reasons for patients declining surgery. Although the results suggest that surgery improves survival in patients with technically operable disease who were offered surgery, patients judged to be unfit for surgery by the MDT and those in whom there were other contributors to symptoms in addition to clot burden were excluded. For the individual patient, factors including age and comorbidities will influence outcome following surgery. How these factors influence the patient's decision requires further research.

\section{Conclusions}

We report results from a large consecutively enrolled registry of patients with CTEPH, and have been able to compare characteristics and have identified predictors of survival in patients who did not undergo surgery despite having technically operable disease. Our data show that survival of patients undergoing PEA is excellent and superior to patients declining surgery, and strongly favours consideration of a surgical intervention in eligible patients. More work is required to understand factors influencing decision making in CTEPH, and to ensure that patients are counselled and supported to make informed decisions. 
Author contributions: S.R. Quadery, A.J. Swift, J.M. Wild, R. Condliffe and D.G. Kiely conceived the idea for the study. S.R. Quadery, A.J. Swift, C.S. Johns, J.M. Wild, C.A. Elliot, J. Hurdman, A.M. Rothman, A. Lawrie, R. Condliffe and D.G. Kiely participated in the study design. S.R. Quadery, A.J. Swift, C.G. Billings, A.A.R. Thompson, C.A. Elliot, J. Hurdman, A. Charalampopoulos, I. Sabroe, I.J. Armstrong, N. Hamilton, P. Sephton, S. Garrod, J. Pepke-Zaba, D.P. Jenkins, N. Screaton, T. Cleveland, S. Thomas, S. Rajaram, C. Hill, C. Davies, C.S. Johns, J.M. Wild, C.A. Elliot, R. Condliffe and D.G. Kiely acquired the data and classified disease. S.R. Quadery, A.J. Swift, C.G. Billings, A.A.R. Thompson, C.S. Johns, J.M. Wild, R. Condliffe and D.G. Kiely analysed and interpreted the data. S.R. Quadery, A.J. Swift, C.G. Billings, A.A.R. Thompson, C.A. Elliot, J. Hurdman, A. Charalampopoulos, I. Sabroe, D.P. Jenkins, A. Lawrie, C.S. Johns, J.M. Wild, R. Condliffe and D.G. Kiely drafted the manuscript. All authors read and revised the content critically, approved the final manuscript, and are accountable for its content.

Conflict of interest: A.A.R. Thompson reports nonfinancial support (travel grants) from Actelion Pharmaceuticals Ltd, outside the submitted work. C.A. Elliot reports personal fees for lecturing and advisory board work from Actelion Pharmaceuticals, GlaxoSmithKline and Bayer, grants for research from Pfizer, Actelion Pharmaceuticals and Bayer, and grants for travel and attendance at conferences from Bayer and Actelion Pharmaceuticals, outside the submitted work. J. Hurdman was part funded as a clinical research fellow by an unrestricted educational grant from Actelion, and has received funding to attend conferences from Actelion, GSK and Pfizer. A. Charalampopoulos has received honoraria for lecturing and taking part in advisory boards, as well as support to attend scientific events/congresses from Actelion, GSK, Servier and MSD. N. Hamilton has and continues to receive honoraria for participation in advisory boards and educational meetings, and has and continues to receive funding to attend educational meetings from a number of pharmaceutical companies including Actelion, Bayer, GSK and MSD (these companies manufacture drug therapies for a variety of indications, but include areas of clinical interest in pulmonary hypertension). J. Pepke-Zaba, or her institution, has received research, educational grants and speaker's honoraria from Actelion, Bayer Pharma AG, Merck and GSK. D.P. Jenkins reports personal fees for lecturing from Bayer, and personal fees for lecturing and consultancy work for the MERIT study from Actelion, outside the submitted work. A. Lawrie reports research grants from the British Heart Foundation, Medical Research Council UK, Actelion Pharmaceuticals and GSK, and conference travel and scientific meeting support from Actelion Pharmaceuticals, outside the submitted work. R. Condliffe has received honoraria for lecturing and advisory boards from Actelion, Bayer and GSK, outside the submitted work. D.G. Kiely has received funding to attend educational meetings, fees for giving educational lectures and participating in advisory boards, and institutional funding for research from Actelion, Bayer and GSK, and has received funding to attend educational meetings, and fees for giving educational lectures and participating in advisory boards from MSD, outside the submitted work.

Support statement: This work was supported by the Wellcome Trust (grant number 205188/Z/16/Z).

\section{References}

1 Kim NH, Delcroix M, Jenkins DP, et al. Chronic thromboembolic pulmonary hypertension. J Am Coll Cardiol 2013; 62: D92-D99.

2 Lang IM, Simonneau G, Pepke-Zaba JW, et al. Factors associated with diagnosis and operability of chronic thromboembolic pulmonary hypertension a case-control study. Thromb Haemost 2013; 110: 83-91.

3 Pepke-Zaba J, Jansa P, Kim NH, et al. Chronic thromboembolic pulmonary hypertension: role of medical therapy. Eur Respir J 2013; 41: 985-990.

4 Hoeper MM, Mayer E, Simonneau G, et al. Chronic thromboembolic pulmonary hypertension. Circulation 2006; 113: 2011-2020.

5 Humbert M. Pulmonary arterial hypertension and chronic thromboembolic pulmonary hypertension: pathophysiology. Eur Respir Rev 2010; 115: 59-63.

6 Lang IM. Chronic thromboembolic pulmonary hypertension - not so rare after all. N Engl J Med 2004; 350: 2236-2238.

7 Moser KM, Braunwald NS. Successful surgical intervention in severe chronic thromboembolic pulmonary hypertension. Chest 1973; 64: 29-35.

8 Jamieson SW, Kapelanski DP, Fedullo PF, et al. Pulmonary endarterctomy: experience and lessons learned in 1500 cases. Ann Thorac Surg 2003; 76: 1457-1462.

9 Cannon JE, Pepke-Zaba J. Is distal chronic thromboembolic pulmonary hypertension treatable with PAH targeted drugs? Semin Respir Crit Care Med 2013; 34: 620-626.

10 Ghofrani HA, D'Armini AM, Grimminger F, et al. Riociguat for the treatment of chronic thromboembolic pulmonary hypertension. N Engl J Med 2013; 369: 319-329.

11 Jais X, D'Armini AM, Jansa P, et al. Bosentan for treatment of inoperable chronic thromboembolic pulmonary hypertension. J Am Coll Cardiol 2008; 52: 2127-2134.

12 Reichenberger F, Voswinckel R, Enke B, et al. Long-term treatment with sildenafil in chronic thromboembolic pulmonary hypertension. Eur Respir J 2007; 30: 922-927.

13 Simonneau G, D'Armini AM, Ghofrani HA, et al. Predictors of long-term outcomes in patients treated with riociguat for chronic thromboembolic pulmonary hypertension: data from the CHEST-2 open-label, randomised, long-term extension trial. Lancet Respir Med 2016; 4: 372-380.

14 Suntharalingam J, Treacy CM, Doughty NJ, et al. Long-term use of sildenafil in inoperable chronic thromboembolic pulmonary hypertension. Chest 2008; 134: 229-236.

15 Feinstein JA, Goldhaber SZ, Lock JE, et al. Balloon pulmonary angioplasty for treatment of chronic thromboembolic pulmonary hypertension. Circulation 2001; 103: 10-13.

16 Mizoguchi H, Ogawa A, Munemasa M, et al. Refined balloon pulmonary angioplasty for inoperable patients with chronic thromboembolic pulmonary hypertension. Circ Cardiovasc Interv 2012; 5: 748-755.

17 Bonderman D, Skoro-Sajer N, Jakowitsch J, et al. Predictors of outcome in chronic thromboembolic pulmonary hypertension. Circulation 2007; 115: 2153-2158.

18 Galie N, Humbert M, Vachiery JL, et al. 2015 ESC/ERS guidelines for the diagnosis and treatment of pulmonary hypertension. Eur Respir J 2015; 46: 903-975.

19 Konstantinides SV. 2014 ESC guidelines on the diagnosis and management of acute pulmonary embolism. Eur Heart J 2014; 35: 3145-3146. 

endarterectomy: results from the United Kingdom national cohort. Circulation 2016; 133: 1761-1771. experience of more than 2,700 patients. Ann Thorac Surg 2012; 94: 97-103.

22 Lewczuk J, Piszko P, Jagas J, et al. Prognostic factors in medically treated patients with chronic pulmonary embolism. Chest 2001; 119: 818-823.

23 Riedel M, Stanek V, Widimsky J, et al. Long term follow-up of patients with pulmonary thromboembolism: late prognosis and evolution of haemodynamic and respiratory data. Chest 1982; 81: 151-158.

24 Delcroix M, Lang I, Pepke-Zaba J, et al. Long-term outcome of patients with chronic thromboembolic pulmonary hypertension: results from an international prospective registry. Circulation 2016; 133: 859-871.

25 Escribano-Subias P, Del Pozo R, Roman-Broto A, et al. Management and outcomes in chronic thromboembolic pulmonary hypertension: from expert centers to a nationwide perspective. Int J Cardiol 2016; 203: 938-944.

26 Pepke-Zaba J, Delcroix M, Lang I, et al. Chronic thromboembolic pulmonary hypertension (CTEPH) results from an international prospective registry. Circulation 2011; 124: 1973-1981.

27 Hurdman J, Condliffe R, Elliot CA, et al. ASPIRE registry: Assessing the Spectrum of Pulmonary hypertension Identified at a REferral centre. Eur Respir J 2012; 39: 945-955.

28 Quadery S, Condliffe R, Billings C, et al. Chronic thromboembolic pulmonary hypertension: long term outcomes in surgical and non-surgical patients. Thorax 2016; 71: A98.

29 Quadery S, Billings C, Wild J, et al. Chronic thrombo-embolic pulmonary hypertension: long-term outcomes in operated and non-operated patients. Eur Respir J 2016; 48: PA3605.

30 Condliffe R, Kiely DG, Gibbs JS, et al. Prognostic and aetiological factors in chronic thromboembolic pulmonary hypertension. Eur Respir J 2009; 33: 332-338.

31 Nierlich P, Hold A, Ristl R. Outcome after surgical treatment of chronic thromboembolic pulmonary hypertension: dealing with different patient subsets. A single-centre experience. Eur J Cardiothorac Surg 2016; 50: 898-906.

32 Skoro-Sajer N, Marta G, Gerges C, et al. Surgical specimens, haemodynamics and long-term outcomes after pulmonary endarterectomy. Thorax 2014; 69: 116-122.

33 Corsico AG, D'Armini AM, Cerveri I, et al. Long-term outcome after pulmonary endarterectomy. Am J Respir Crit Care Med 2008; 178: 419-424.

34 Saouti N, Morshuis WJ, Heijmen RH, et al. Long-term outcome after pulmonary endarterectomy for chronic thromboembolic pulmonary hypertension: a single institution experience. Eur J Cardiothorac Surg 2009; 35: 947-952.

35 Condliffe R, Kiely DG, Gibbs JS, et al. Improved outcomes in medically and surgically treated chronic thromboembolic pulmonary hypertension. Am J Respir Crit Care Med 2008; 177: 1122-1127.

36 Thakrar MV, Hall A, Crackett R, et al. Functional and quality of life improvements in treated patients with chronic thromboembolic pulmonary hypertension. J Heart Lung Transplant 2013; 32: S304.

37 Genta P, Jatene F, Terra-Filho M. Quality of life before and after pulmonary thromboendarterectomy: preliminary results. J Bras Pneumol 2005; 31: 48-51.

38 Harris C, Jenkins M, Glaser D. Gender differences in risk assessment: why do women take fewer risks than men? Judgment Decis Mak 2006; 1: 48-63.

39 D'Armini AM, Morsolini M, Mattiucci G, et al. Pulmonary endarterectomy for distal chronic thromboembolic pulmonary hypertension. J Thorac Cardiovasc Surg 2014; 148: 1005-1011.

40 Mayer E, Jenkins D, Lindner J, et al. Surgical management and outcome of patients with chronic thromboembolic pulmonary hypertension: results from an international prospective registry. J Thorac Cardiovasc Surg 2011; 141: $702-710$ 